\title{
Cashew apple juice as microbial cultivation medium for non-immunogenic hyaluronic acid production
}

\author{
Adriano H. Oliveira ${ }^{1}$, Cristiane C. Ogrodowski ${ }^{2}$, André C. de Macedo ${ }^{1}$, \\ Maria Helena A. Santana ${ }^{2}$, Luciana R.B. Gonçalves ${ }^{1}$ \\ ${ }^{1}$ Departamento de Engenharia Química, Universidade Federal do Ceará, Fortaleza, CE, Brazil. \\ ${ }^{2}$ Faculdade de Engenharia Química, Universidade Estadual de Campinas, Campinas, SP, Brazil.
}

Submitted: March 1, 2012; Approved: April 4, 2013.

\begin{abstract}
In this work, natural cashew apple juice was used as cultivation medium as an alternative to substitute brain heart infusion medium. The effect of aeration and juice supplementation with yeast extract on the production of hyaluronic acid in batch fermentation was also investigated. Similar levels of cell mass were obtained in inoculum using cashew apple juice supplemented with yeast extract or the conventional brain heart infusion medium. Fermentation in Erlenmeyer flasks produced low biomass and hyaluronic acid concentrations. The hyaluronic acid concentration and viscosity increased from $0.15 \mathrm{~g} / \mathrm{L}$ and $3.87 \mathrm{cP}$ (no aeration or medium supplementation) to $1.76 \mathrm{~g} / \mathrm{L}$ and $107 \mathrm{cP}$, when aeration $(2 \mathrm{vvm})$ and $60 \mathrm{~g} / \mathrm{L}$ of yeast extract were used. The results suggest the production of low-molecular weight hyaluronic acid oligomers instead of the high molecular weight polymer.
\end{abstract}

Key words: hyaluronic acid, cashew apple juice, submerged fermentation.

\section{Introduction}

Hyaluronic acid (HA), also commercially referred to as hyaluronan, is a linear high molecular weight glycosaminoglucan polysaccharide, composed by D-glucuronic acid and $\mathrm{N}$-acetylglucosamine, which exists in many mammalian connective tissues such as joints, vitreous bodies, umbilical cords, cartilages, skins, and combs of fowls as a constituent (Yamada and Kawasaki, 2005). In rooster comb, for instance, HA is complexed with proteoglycans and often contaminated with HA degrading enzymes, making the isolation of high purity and high molecular weight HA very difficult and costly (Blank et al., 2005). Furthermore, the risk of cross-species viral and other infection agent has been pointed out when using animal-derived biochemicals for human therapeutics (Blank et al., 2005; Yamada and Kawasaki, 2005).

As an alternative, HA also can be produced by grampositive bacterium Streptococcus equi subspecies zooepidemicus, which is generally the strain used in industry since it synthesizes HA as an extracellular capsule (Patil et al., 2011). Microbial HA is non-immunogenic and chemi- cally identical to mammalian polysaccharide. Consequently it is regarded as a viable substitute for the HA currently derived from other sources (Chong and Nielsen, 2003). The microbial HA production is becoming more and more interesting due to the possibility of process control and optimization, therefore, achieving higher product yields. In addition, the main advantage of the fermentative over traditional process, umbilical cords extraction, for instance, is the attainment of a product free of antigens. Other advantages include less laborious purification steps and achievement of a high molecular mass HA (Swann et al., 1990; Ellwood et al., 1996).

Streptococci strains used in HA production are fastidious with respect to their nutrient and organic nitrogen requirements (Hofvendahl and Hahn-Hägerdal, 2000; Fitzpatrick and Keeffe, 2001). Natural resources have been recently studied aiming to obtain low-cost cultivation media and a better purity grade of HA. Pires et al. (2010) evaluated different agricultural resource derivatives (ARDs), such as hydrolysate soy protein concentrate, whey protein concentrate, and cashew apple juice aiming at the production of HA by Streptococcus zooepidemicus. Corn steep li- 
quor was also evaluated as a supplement. Among the studied ARDs, only cashew apple juice supplemented with yeast extract (CAJY) showed to be a promising medium due to the presence of complex nutrients, such as B-vitamins, particularly thiamine, riboflavin, niacin and folic acid (Sancho et al., 2007). HA produced in CAJY presented an average molecular weight of $104 \mathrm{Da}$, which finds applications in biomedical and healthcare fields (Pires et al., 2010). Likewise submerged fermentation of the cashew apple juice, the production of HA by solid-state fermentation of the juice-moisturized cashew apple bagasse was found to be significant (Macedo and Santana, 2012). The authors achieved a yield of $761.25 \mathrm{mg}$ HA per kilo of total cashew apple fruit, which shows that HA production from the total cashew apple pseudo-fruit is promising and economically viable. Additionally, cashew is produced in 32 countries in the world and Brazil is one of the major cashew appleproducing countries. The production figures for the year of 2010, based on the Food and Agriculture Organization, are 104,342 tons (FAO, 2010). However, only $18 \%$ of the total peduncle is exploited for obtaining various products, from concentrated juice to desserts, and $80 \%$ of the pulp is wasted (Oliveira et al., 2013).

Likewise the nutrient requirements, oxygen transfer also plays an important role on the HA production by submerged cultivation. In the presence of oxygen, $S$. zooepidemicus grows faster and has a higher biomass yield. Furthermore, HA yield and molecular weight are higher when compared to anaerobic cultivation (Chong and Nielsen, 2003). Under aerobic conditions, glucose uptake and growth rates are increased and, as expected; more acetate was formed with little or no ethanol and formate production. This altered metabolism enhances HA productivity and molecular weight (Johns et al., 1994; Nielsen, 2005; Ogrodowski et al., 2005).

Considering the above mentioned aspects, in this work, cashew apple juice, here named CAJ, was used as culture medium for HA production using S. zooepidemicus. Since the biopolymer can be produced anaerobically or aerobically, the effect of aeration on biomass production and HA concentration was investigated. Furthermore, the effect of medium supplementation with yeast extract as a substitute for the conventional medium Brain and Heart infusion (BHI) on the final HA concentration was also studied.

\section{Material and Methods}

\section{Cashew apple juice (CAJ) preparation}

Cashew apple juice (CAJ) was used as medium for inoculum preparation and batch fermentation for HA production due to its rich composition (Rocha et al., 2007) that includes $10 \%$ of reducing sugars (glucose and fructose), phosphorous and amino acids. The juice was withdrawn by compressing the cashew apple (Anarcardium occidentale $L$.). Afterwards, $\mathrm{pH}$ was adjusted to 7.0 and the medium was submitted to ultraviolet exposition for $2 \mathrm{~h}$ for sterilization in order to avoid loss of labile components by heat.

\section{Microorganism and inoculum preparation}

Streptococus zoopedemicus (ATCC 39920) was obtained from the American Type Culture Collection as a lyophilized culture in ampoules and it was maintained on Trypticase Soy Agar (TSA) at $5{ }^{\circ} \mathrm{C}$. The inoculum was incubated at $37^{\circ} \mathrm{C}$ on a rotary shaker at $150 \mathrm{rpm}$ for $48 \mathrm{~h}$ in a $500 \mathrm{~mL}$ Erlemeyer flask containing $200 \mathrm{~mL}$ of medium. Natural cashew apple juice (CAJ), CAJ supplemented with $37.5 \mathrm{~g} / \mathrm{L}$ of BHI and CAJ supplemented with $60 \mathrm{~g} / \mathrm{L}$ of yeast extract, here named CAJY, were used for inoculum preparation. Conventional BHI medium was used as control. The cells were harvested by centrifugation and were used latter to inoculate the fermentation broth.

\section{Medium and fermentation conditions}

Cashew apple juice and CAJ supplemented with $60 \mathrm{~g} / \mathrm{L}$ of yeast extract were used in batch fermentations. The inoculum concentration was $10 \% \mathrm{v} / \mathrm{v}$ and the fermentation was conducted at $37{ }^{\circ} \mathrm{C}$ and $150 \mathrm{rpm}$. First, assays were carried out in $500 \mathrm{~mL}$ Erlenmeyers flasks containing $200 \mathrm{~mL}$ medium. After, to investigate the effect of aeration, a 3 L BioFLO III fermenter (New Brunswick Scientific Co. Inc., Edison, NJ, USA) with an operating volume of $2.0 \mathrm{~L}$ and $2 \mathrm{vvm}$ aeration was used. The culture $\mathrm{pH}$ in the fermenter was controlled at 7.0 by automatic addition of sodium hydroxide solution.

\section{Hyaluronic acid purification}

The separation and purification of HA was performed by precipitation with ethanol according to Brown et al. (1988). Cells were removed from culture broth by centrifugation and the supernatant was treated with ethanol in a proportion 3:2(v/v) ethanol:supernatant. The solution remained at rest for the precipitation of HA. The precipitate was recovered by centrifugation at $3000 \mathrm{rpm}$ for $20 \mathrm{~min}$ and redissolved in $\mathrm{NaCl} 0.15 \mathrm{M}$. In order to increase the yield of HA, three steps of precipitation and redissolution were performed (Pires et al., 2010).

\section{Analytical methods}

Biomass concentration was monitored by measuring the dry weigh $(\mathrm{g} / \mathrm{L})$. Samples of culture medium (approx. $100 \mathrm{~mL}$ ) were centrifuged at $3000 \mathrm{rpm}$ for $20 \mathrm{~min}$. The harvested cells were then dried to constant weight at $60^{\circ} \mathrm{C}$ to determine the dry weight content of the medium. Relatively higher values of dry weight measurements might be obtained due to interference from the presence of capsular HA, which might not be removed before analysis. This interference is more pronounced with aerated cultures, since cells under such conditions tend to possess very large capsules (Cooney et al., 1999). The total reducing sugars concentration was determined according to Somogy-Nelson 
method (Somogyi, 1952). Hyaluronic acid concentration in the culture broth, measured as sodium hyaluronate, was determined by high performance liquid chromatography (Johns et al., 1994), using a refractive index detector (Waters 410). Filtered broth samples were injected into a HPLC equipped with OHPak SB-806 HQ column, working at $40{ }^{\circ} \mathrm{C}$. The mobile phase consisted of $10 \mathrm{mM} \mathrm{NaNO}_{3}$ solution pumped at a flow rate of $1 \mathrm{~mL} / \mathrm{min}$. The standard curve was prepared using sodium hyaluronate (Hylumed@ Genzyme Corporation). The viscosity of the produced sodium hyaluronate was determined using a Haake rheometer CV20 with parallel plate modules. All measurements were performed in triplicate and results represent the means.

\section{Yield and Kinetic parameters}

The yields in product $\left(Y_{P / S}\right)$ and cells $\left(Y_{X / S}\right)$ related to substrate, and the specific rates for growth, $\mu_{X}$ and product formation $\mu_{P}$, were calculated from the measured concentrations of product, substrate and cells. HA productivity $\left(P_{P}\right)$ was calculated as the ratio of maximum product concentration $P_{m}$ to the fermentation time, see Eq.1, and biomass productivity $\left(P_{X}\right)$ was calculated as the ratio of $\left(X_{m}-\right.$ $\left.X_{0}\right)$ to the fermentation time, see Eq. 2

$$
\begin{aligned}
& P_{p}=\frac{P_{m}}{t_{f m}} \\
& P_{x}=\frac{X_{m}-X_{0}}{t_{f}}
\end{aligned}
$$

where $X_{0}$ is initial biomass concentration, $X_{m}$ is the maximum biomass concentration after a fermentation time $t_{f} . P_{m}$ is the maximum product concentration after a fermentation time $t_{f m}$.

\section{Results and Discussion}

\section{Effect of culture medium on cell growth and inoculum preparation}

Optimum culture conditions for HA production in batch mode is well described in the literature (Armstrong and Kim et al., 1996; Johns, 1997; Stangohl, 2000; Chong et al., 2005). Blood or serum is usually described as essential for colony growth and development in the strains of group C Streptococcus (Chong et al., 2005). However, there is a potential risk for blood contamination (Zhang et al., 2006). Therefore, in this work a different serum-free media for cell growth was studied and results were compared to a medium prepared using BHI (control).

Figure 1 shows the effect of culture medium on $S$. zooepidemicus growth during inoculum preparation. It can be observed that every medium induced colony growth and development, but low cell concentration $(7.8 \mathrm{~g} / \mathrm{L})$ was obtained when pure CAJ was used. Enriching CAJ with $37 \mathrm{~g} / \mathrm{L}$ BHI or using CAJY medium yielded similar bio-

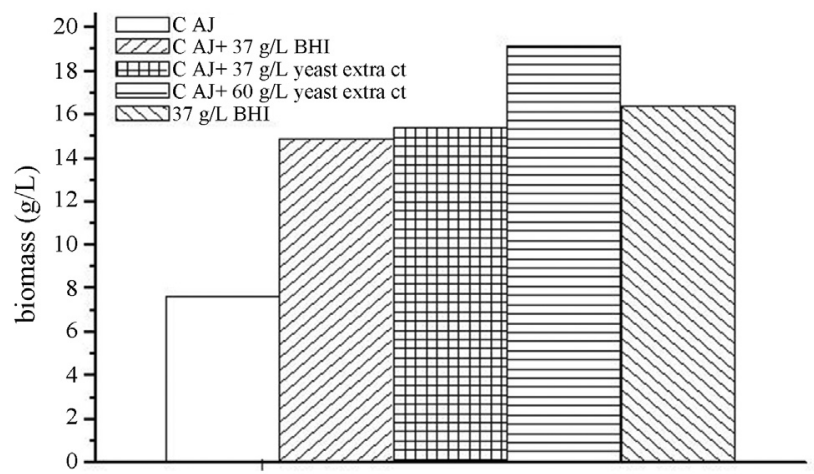

Figure 1 - Comparative performance of culture media for S. zooepidemicus groth: Crude cashew apple juice, CAJ, crude cashew apple juice, $\mathrm{CAJ}$ with $37 \mathrm{~g} / \mathrm{L}$ or $60 \mathrm{~g} / \mathrm{L}$ yeast extract, CAJ with $37 \mathrm{~g} / \mathrm{L}$ Brain Heart Infusion, $\mathrm{BHI}$ and $\mathrm{BHI}$ medium $37 \mathrm{~g} / \mathrm{L}$.

mass concentrations. The highest cell concentration was produced in CAJY as compared to the other media, including the control.

Lancefield group A and C streptococci bacteria require complex nutrients due to their limited ability to synthesize specific amino acids and B-vitamins (Hofvendahl and Hahn-Hägerdal, 2000; Fitzpatrick and Keeffe, 2001). Additionally to this fact, there is the nutritional requirement with respect to organic nitrogen, which also supplies a large proportion of carbon for the cellular biosynthesis (Armstrong and Johns, 1997). As reported in the literature, CAJ has low total nitrogen content $(0.04 \% \mathrm{w} / \mathrm{v})$ and requires an additional nitrogen supplementation to reach an ideal glucose/nitrogen ratio (Pires et al., 2010). When yeast extract was used to enrich CAJ (CAJY), a favorable cultivation condition was established by the presence of purine and pirimidine bases and the B-vitamins, which are essential to the streptococci cultivation. The CAJ also contains B-vitamins, mainly thiamin, riboflavin, niacin and folic acid, which are beneficial to the streptococci cultivation (Amrane and Prigent, 1994; Sancho et al., 2010).

The yeast extract that was added to CAJ was able to create a suitable condition for Streptococcus zooepidemicus growth. These results demonstrate that the conventional BHI medium can be advantageously substituted by CAJY medium for inoculum preparation.

\section{Influence of medium supplementation with yeast extract and aeration on hyaluronic acid production}

The kinetic profiles in Figure 2 ( $A$ and $B$ ) describe the average behavior of fermentations carried out in Erlenmeyer flasks with CAJ and CAJY media. When CAJ medium was used, sugars were starved from medium in $16 \mathrm{~h}$ remaining at a low level (approximately $0.05 \mathrm{~g} / \mathrm{L}$ ), Figure $2 \mathrm{~A}$. Similar but faster $(4 \mathrm{~h})$ uptake of sugars was observed on CAJY medium. Analyzing the data, it can be observed that the presence of essential nutrients in CAJY increased cell growth and HA production, when compared to the CAJ. Biomass and HA production were 3 -fold higher 

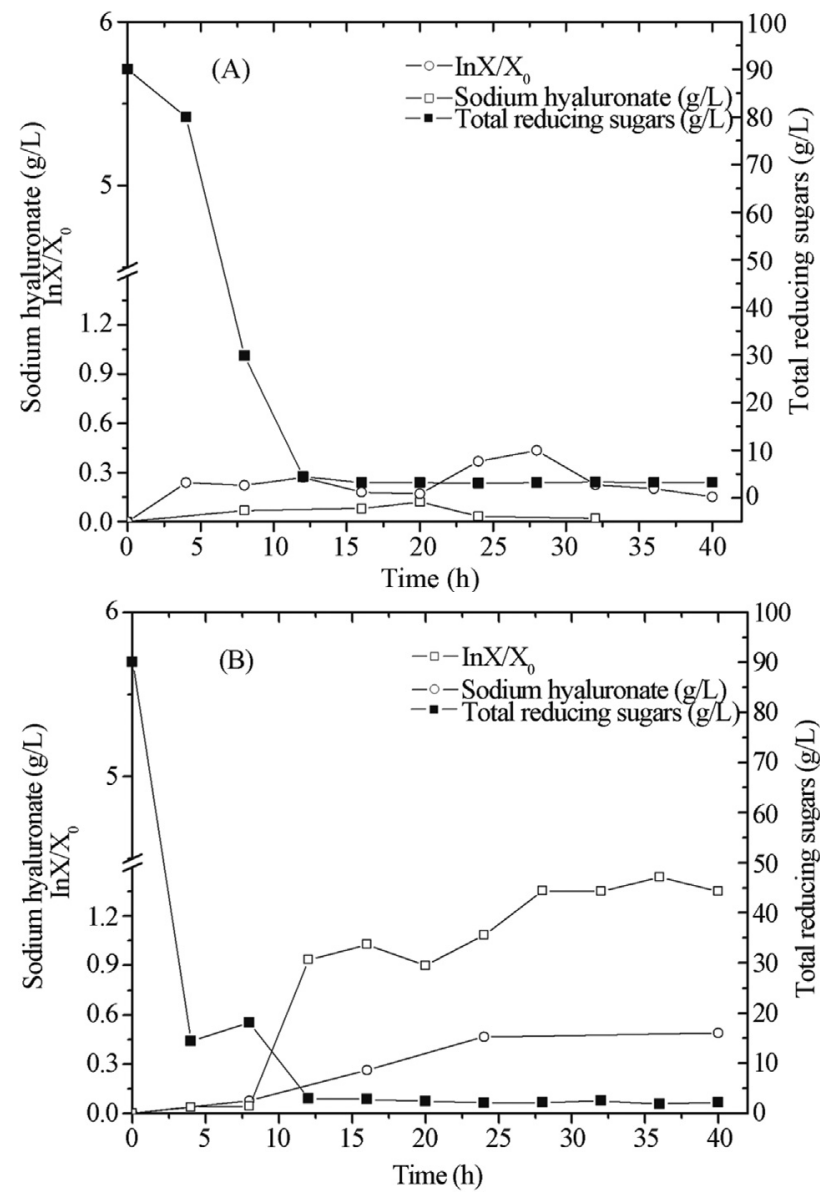

Figure 2 - Kinetic profiles of biomass ( $\left.\ln \mathrm{x} / \mathrm{x}_{0}\right)$, reducing sugars and hyaluronic acid for fermentation using $S$. zooepidemicus and natural aeration (Erlenmeyer flasks) (A) crude cashew apple juice, CAJ (B) crude cashew apple juice enriched with $60 \mathrm{~g} / \mathrm{L}$ yeast extract, CAJY.

on CAJY medium (Figure 2B). A diauxic behavior could also be observed, in CAJ medium, when a second exponential phase appeared after $20 \mathrm{~h}$, with a concomitant decrease in HA concentration (Figure 2A).

The $\mathrm{pH}$ variation during $S$. zooepidemicus growth in $\mathrm{CAJ}$ and CAJY medium (Figure 3) was also compared. It can be observed that at the begging of the essay, $\mathrm{pH}$ decreased in both CAJ and CAJY medium. From 5 to $15 \mathrm{~h}$, while $\mathrm{pH}$ of CAJ continued to decrease, $\mathrm{pH}$ of CAJY remained almost constant. After $15 \mathrm{~h}$, the $\mathrm{pH}$ of CAJ started to increase, while $\mathrm{pH}$ of CAJY decreased again. According to the literature (Armstrong and Johns, 1997), lactic acid is the main metabolite arising from glucose catabolism in $S$. zooepidemicus, which explains the decrease in $\mathrm{pH}$ values observed for both mediums. The fact that $\mathrm{pH}$ values decrease earlier in CAJ medium compared to CAJY may suggest that more lactic acid is produced in CAJ medium. This decreased synthesis of lactic acid in CAJY favored strain growth, as observed by other authors (Zhang et al., 2006) when studying serum-free medium for colony growth and hyaluronic acid production by Streptococcus zooepi-

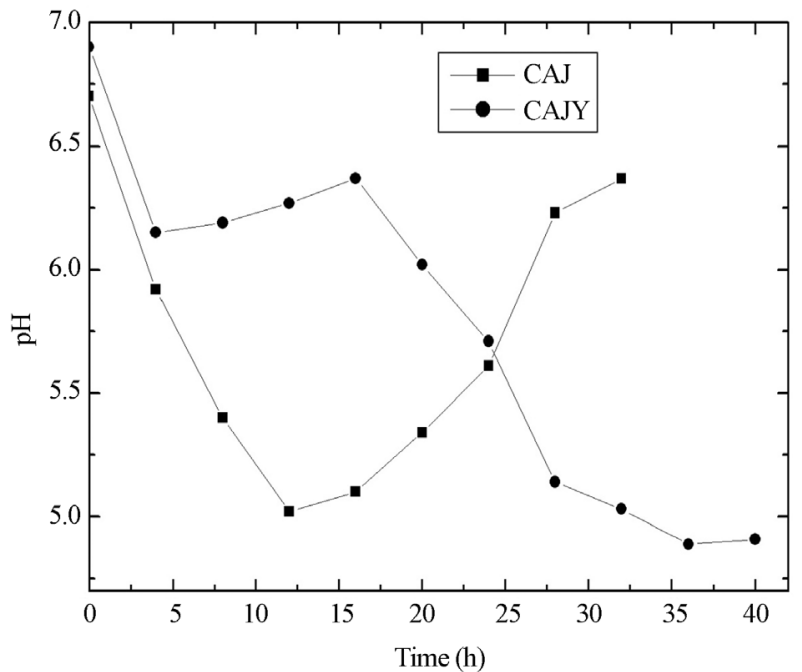

Figure 3 - Variation of $\mathrm{pH}$ during fermentation of CAJ and CAJY using $S$. zooepidemicus and natural aeration (Erlenmeyer flasks).

demicus NJUST01. It is known from the literature (Liu et al., 2011) that lactic acid accumulation causes a strong inhibition of cell growth and HA.

Based on cell growth, HA production and $\mathrm{pH}$ values observed for both mediums, CAJ supplementation with yeast extract was favorable for HA synthesis under natural aeration. Nevertheless, it is well known that microbial production of HA is significantly influenced by culture conditions, such as aeration rate, dissolved oxygen concentration and bioreactor type (Swann et al., 1990; Nielsen, 2005; Liu et al., 2011). Therefore, the effect of aeration and medium supplementation on HA production were investigated in a 3 L BioFLO III fermenter with an operating volume of $2.0 \mathrm{~L}$ at $150 \mathrm{rpm}, 37^{\circ} \mathrm{C}$ and $2 \mathrm{vvm}$ aeration. Figure 4 shows HA production, cell growth and substrate uptake under aerobic conditions. Table 1 compares the influence of aeration and juice supplementation on sodium hyaluronate production. It can be observed that the juice supplementation under aerobic conditions was not beneficial since HA concentration decreased from $1.88 \mathrm{~g} / \mathrm{L}$, when pure juice was used (Figure $4 \mathrm{~A}$ ), to almost $1.76 \mathrm{~g} / \mathrm{L}$, when yeast extract was added to the juice (Figure $4 \mathrm{~B}$ ). Aeration plays a more pronounced effect on sodium hyaluronate production, since the sodium hyaluronate concentration increased from $0.12 \mathrm{~g} / \mathrm{L}$ to $1.88 \mathrm{~g} / \mathrm{L}$ in CAJ. The higher HA concentrations obtained under aerobic conditions is probably caused by a large energetic yield, since, in the presence of oxygen, there is a deviation from pyruvate to acetate rather than to lactate (Swann et al., 1990). In other words, in the presence of dissolved oxygen, the carbon flux towards lactic acid to acetic acid is redirected and more ATP is generated. This extra ATP is used to achieve higher HA titer (Liu et al., 2011). Another explanation for the effects of aeration is that HA is produced in the microbial surroundings to protect themselves against the toxic effects provided by oxygen, espe- 

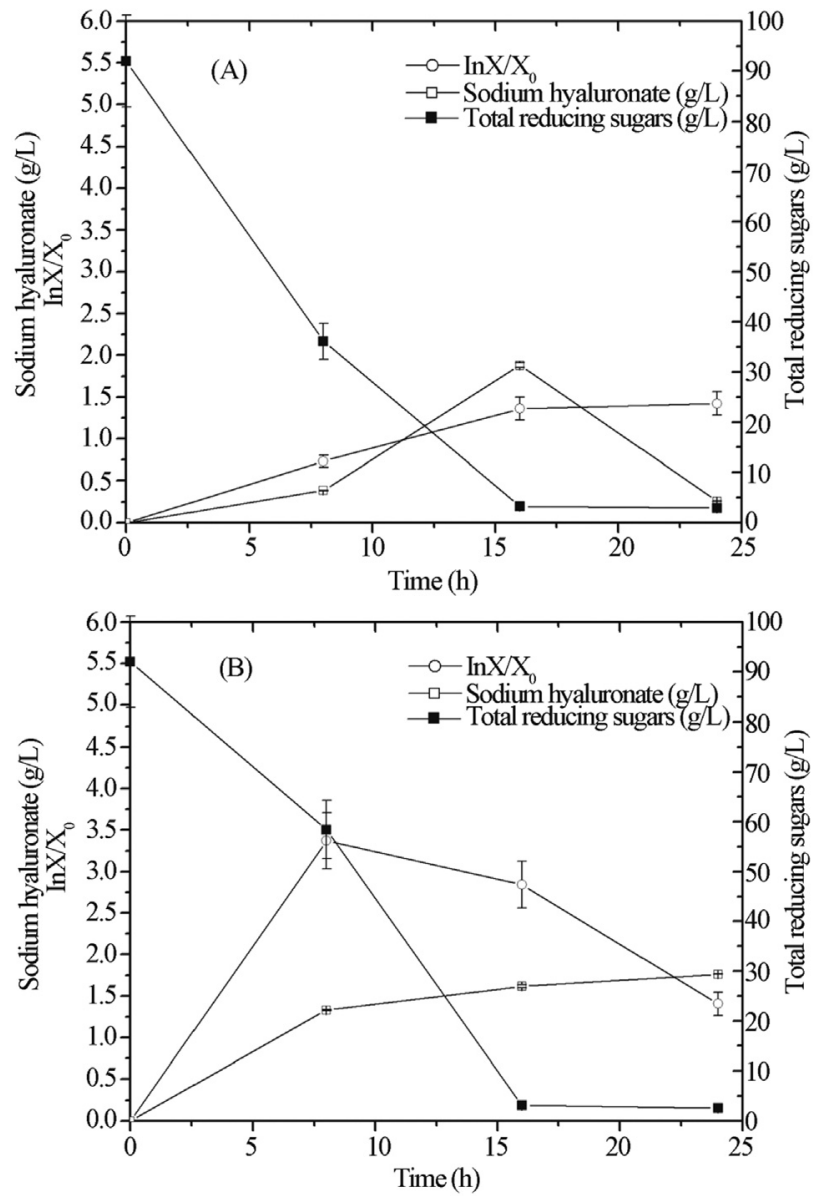

Figure 4 - Kinetic profiles of biomass $\left(\ln \mathrm{x} / \mathrm{x}_{0}\right)$, reducing sugars and hyaluronic acid for fermentation using $S$. zooepidemicus with 2 vvm aeration using: (A) crude cashew apple juice, (B) CAJ crude cashew apple juice enriched with $60 \mathrm{~g} / \mathrm{L}$ yeast extract, CAJY. All assays were performed in duplicate and results represent the means \pm standard deviation.

cially those from the univalent reduction of molecular oxygen and hydroxyl free radicals conditions (Swann et al., 1990; Ellwood et al., 1996; Nielsen, 2005).

In addition, the presence of glucose and fructose as main sugars in CAJ also may have an important role on the HA synthesis due to the metabolic route of glucose conversion to HA. Under limiting aeration (Erlenmeyer flasks), the biosynthesis needs were provided by the complex nitrogen source added to the medium (Pires and Santana, 2010), which explains the 4-fold enhancement in AH concentration when yeast extract was added to CAJ (Table 1). Nevertheless, under forced aeration (BioFLO III fermenter), oxygen was available to complete sugar oxidative metabolic processes and, therefore, medium supplementation with yeast extract did not promote an increase in HA concentration.

Figures 5 and 6 show the effects of aeration and juice supplementation on the specific rates of cell growth and product formation along fermentation. In aerated conditions, the HA production rate is associated to the cell growth rate until $8 \mathrm{~h}$ fermentation. Afterwards, HA and cells compete for energy and metabolic intermediates, and in the supplemented medium, the HA production rate increases at the expenses of decline of cell growth rate. Indeed, it is reported in the literature that glycolysis and HA synthesis compete for the carbon flux (Liu et al., 2011). In other words, a reduction in the rate of biomass formation is effective for the enhancement of HA production, as observed in this work.

Table 2 resumes the kinetic parameters estimated from the experimental data showed on Figures 2 and 3. Under anaerobic conditions, $Y_{P / S}, P_{P}$ and $\mu_{P \max }$ were higher on CAJ, while $P_{x}$ and $\mu_{\text {Xmax }}$ were higher on CAJY. Under aerobic conditions, maximum specific growth rate $\left(\mu_{X \max }\right)$ was much lower on CAJ $\left(0.09 \mathrm{~h}^{-1}\right)$ as compared with CAJY $\left(0.42 \mathrm{~h}^{-1}\right)$. The slower growth rate in CAJ might be explained by the mechanism of amino acid uptake. Certain amino acids compete for the same transport system. The presence of peptide in the yeast extract, used to compose CAJY, allows alternative routes for amino acid uptake (Chong et al., 2005). Furthermore, peptides transport can be more efficient that the transport of individual amino acids (Guirard and Snell, 1962). Maximum specific HA production rate $\left(\mu_{\text {pmax }}\right)$ was also lower on CAJ $\left(0.007 \mathrm{~h}^{-1}\right)$ as compared to CAJY $\left(0.009 \mathrm{~h}^{-1}\right)$, under aerobic conditions.

Maximum specific growth rate $\left(\mu_{X \max }\right)$ CAJY was lower without aeration $\left(0.06 \mathrm{~h}^{-1}\right)$ as compared to the assay when 2 vvm of aeration $\left(0.42 \mathrm{~h}^{-1}\right)$ was used, keeping all other conditions constant. The same behavior is also observed for maximum specific HA production rate, see Table 2. It can also be observed that higher HA $\left(Y_{P / S}\right)$ and growth yields $\left(Y_{X / S}\right)$, HA $\left(P_{P}\right)$ and biomass productivities $\left(P_{X}\right)$ were obtained under aeration and using CAJY. Similar results were obtained by different authors (Kim et al., 1996; Armstrong and Johns, 1997; Chong et al., 2005) when using a chemically defined media. As mentioned before, higher HA production is achieved in an aerobic culture when compared with an anaerobic culture, which may be

Table 1 - Comparative performance of culture media for sodium hyaluronate production without aeration or with 2 vvm aeration: crude cashew apple juice, CAJ, crude cashew apple juice enriched with $60 \mathrm{~g} / \mathrm{L}$ yeast extract, CAJY.

Fermentation media
Sodium hyaluronate concentration $(\mathrm{g} / \mathrm{L})$

Without aeration

0.12

0.49

$1.88 \pm 0.03$

Cashew Apple Juice (CAJ) 


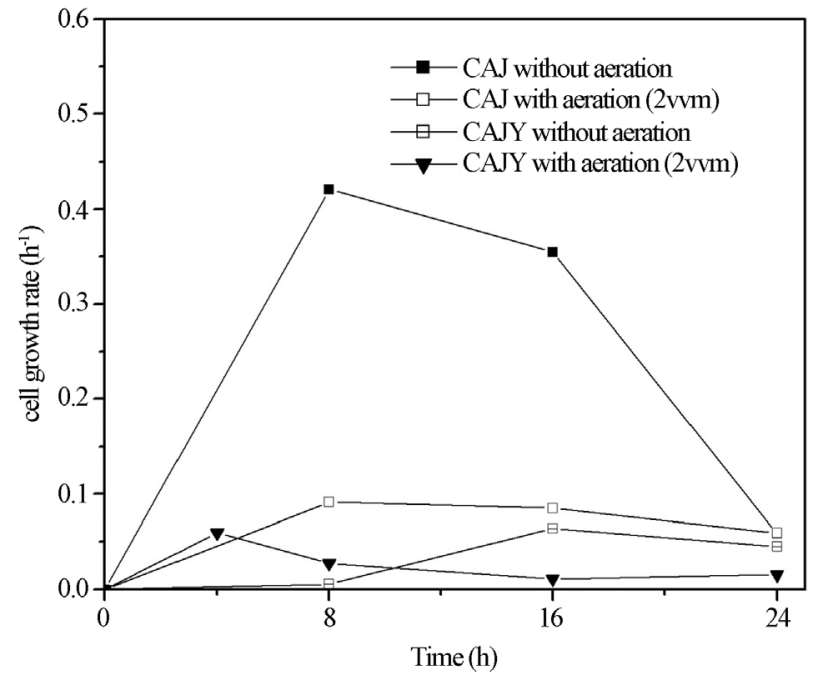

Figure 5 - Specific rate of cell growth of S. zooepidemicus without aeration or with 2 vvm aeration using: crude cashew apple juice (CAJ) or cashew apple juice enriched with $60 \mathrm{~g} / \mathrm{L}$ yeast extract (CAJY).

explained by: (i) redirection of the carbon flux towards lactic acid to acetic acid in the presence of oxygen, which provides more ATP; (ii) oxygen may promote the synthesis of $\mathrm{AH}$ since cells aggregation may protect them from oxygen metabolites; (iii) acetyl-CoA accumulation may be enhanced by aeration, which can be deviated from the carbon metabolism in order to supply the synthesis of AH (Liu et al., 2011).

\section{Rheological characterization}

The viscous behavior of the product obtained by fermentation using CAJY medium in Erlenmeyers flasks or in a 3 L BioFLO III fermenter (with aeration) was investigated. It was observed that culture aeration (viscosity varied from $3.87 \mathrm{cP}$ without aeration to $107.00 \mathrm{cP}$ with $2 \mathrm{vvm}$ aeration), together with supplementation of medium with yeast extract (viscosity varied from $73.27 \mathrm{cP}$ to to $107.00 \mathrm{cP}$ ), caused a positive influence on the HA viscosity. The viscous behavior indicates a low molecular weight $\mathrm{HA}$, less than $500 \mathrm{kDa}$, according to the rheological parameters obtained by other authors (Prieto et al., 2005) for similar concentration of commercial HA. Although precursor compounds were produced, the HA was not polymerized. The production of HA by $S$. zooepidemicus is due to con-

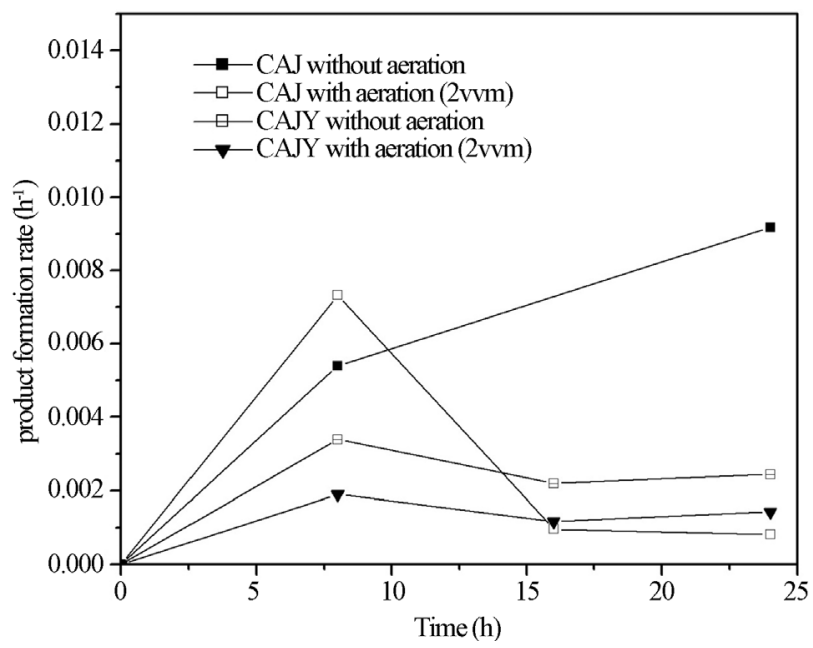

Figure 6 - Specific rate of product formation for fermentation using $S$. zooepidemicus without aeration or with 2 vvm aeration using: crude cashew apple juice (CAJ) or cashew apple juice enriched with $60 \mathrm{~g} / \mathrm{L}$ yeast extract (CAJY).

version of glucose and fructose-6-phosphate in the precursors glucuronic acid and $\mathrm{N}$-acetylglucosamine. The biopolymer is formed by reaction of the precursors mediated by a hyaluronidase synthase (HAS). An abortive translocation model proposed by Forsee et al. (2000) for polymerization of type 3 capsule in Streptococcus pneumoniae by a glycosyltransferase similar to HAS says that in the absence, or at low concentrations, of one of the substrates, while at normal levels of the other substrate, membranebound polymer chains were released into solution. Although this mechanism has not been observed directly for HA polymerization, it seems to explain the data obtained using CAJY as a fermentation medium. The absence of extended polymerization could be due to the presence of some inhibitions in CAJY broth, which unbalanced the HA precursors produced. The absence of the required balance produced low molecular weight HA.

A possible synergic redox-depolymerization mechanism provides by ascorbate presence in cashew apple juice possibly causes HA molecular weight reduction. oltés et al. (2006) and Liu et al. (2009), investigated the reduction of HA molecular weight due to depolymerization, by adding to a high molecular weight HA solution, or hydrogen peroxide and ascorbate along submerged fermentations with $S$.

Table 2 - Parameters obtained for fermentation using S. zooepidemicus, crude cashew apple juice (CAJ) or crude cashew apple juice enriched with $60 \mathrm{~g} / \mathrm{L}$ yeast extract (CAJY), without aeration or under $2 \mathrm{vvm}$ aeration.

\begin{tabular}{lcccccc}
\hline Medium/parameter & $Y_{P / S}$ & $Y_{X / S}$ & $P_{P}\left(\mathrm{~g} . \mathrm{L}^{-1} \cdot \mathrm{h}^{-1}\right)$ & $P_{x}\left(\mathrm{~g} . \mathrm{L}^{-1} \cdot \mathrm{h}^{-1}\right)$ & $\mu_{X \max }\left(\mathrm{h}^{-1}\right)$ & $\mu_{P \max }\left(\mathrm{h}^{-1}\right)$ \\
\hline CAJ $\left(^{*}\right)$ & 0.001 & 0.01 & 0.008 & 0.03 & 0.01 & 0.002 \\
CAJY $(*)$ & 0.006 & 0.01 & 0.019 & 0.24 & 0.06 & 0.002 \\
CAJ $\left(^{* *}\right)$ & 0.021 & 0.07 & 0.118 & 0.57 & 0.09 & 0.06 \\
CAJY $(* *)$ & 0.039 & 0.38 & 0.176 & 3.72 & 0.42 & 0.06 \\
\hline
\end{tabular}

* Without aeration. ** with 2 vvm aeration. 
zooepidemicus in synthetic medium. The authors described the basic mechanism for redox depolymerization, involving the appearance of reactive oxygen species (ROS) that are capable of breaking the HA into smaller molecules.

\section{Conclusion}

The results obtained at this work show that cashew apple juice is a suitable substrate for the growth of Streptococus zooepidemicus and the production of oligomers of HA. Furthermore, cashew apple juice supplemented with yeast extract is a proper substitute for the conventional medium BHI used for inoculum propagation, which is expensive and has a risk for contamination. It was observed that the composition of the culture medium as well as oxygen supply influenced the microbial production of HA (concentration and viscosity), highest concentrations were obtained when the fermentation was carried out under aeration. The hyaluronic acid viscosity increased when cashew apple juice was supplemented with $60 \mathrm{~g} / \mathrm{L}$ of yeast extract. The potentialities of CAJY medium are evident, with the advantages that BHI did not need to be added, resulting in a fermentation medium without risk of cross-species viral and other infection agent. These results point out to a straight and safe process to produce HA oligomers suitable to important medical applications as a high value-added product. Nowadays, most of methods for production of HA oligomers involve enzymatic digestion or sonication of polymeric HA followed by purification of fractions.

\section{Acknowledgments}

The authors acknowledge FINEP, CNPq, CAPES, FAPESP and FUNCAP for the financial support that made this work possible.

\section{References}

Amrane A, Prigent Y (1994) Lactic acid production from lactose in batch culture: analysis of the data with the help of a mathematical model; relevance for nitrogen source and preculture assessment. Appl Microbiol Biotechnol 40:644-649.

Armstrong DC, Johns MR (1997) Culture conditions affect the molecular weight properties of hyaluronic acid produced by Streptococus zoopidemicus. Appl Environ Microbiol 63:2759-2764.

Blank LM, McLaughlin RL, Nielsen LK (2005) Stable Production of Hyaluronic Acid in Streptococcus zooepidemicus chemostats operated at high dilution rate. Biotechnol Bioeng 90:685-693.

Brown KK, Ruiz LC, Van de Rijn I. 1988. Ultrapure hyaluronic acid and method of making it. US Pat. 4,782,046.

Chong BF, Blank LM, McLaughlin R, Nielsen LK (2005) Microbial Hyaluronic acid production. Appl Microbiol Biotechnol 66:341-351.

Chong BF, Nielsen LK (2003) Aerobic cultivation of Streptococcus zooepidemicus and the role of NADH oxidase. Biochem Eng J 16:153-162.
Cooney MJ, Goh LT, Lee PL, Johns MR (1999) Structured Model-based analysis and control of the Hyaluronic Acid Fermentation by Streptococcus zooepidemicus, Physiological implications of glucose and complex-nitrogen limited growth. Biotechnol Prog 15:898-910.

Ellwood DC, Evans CGT, Dunn GM, McInnes N, Yeo RG, Smith KJ. 1996. Production of hyaluronic acid. U.S. Pat. $5,563,051$.

FAO, http://faostat.fao.org/site/339/default.aspx, accessed in 11/12/2012.

Fitzpatrick JJ, O'Keeffe U (2001) Influence of whey protein hydrolyzate addition to whey permeate batch fermentations for producing lactic acid. Proc Biochem, 37:183-186.

Forsee WT, Carter RT, Yother J (2000) Biosynthesis of type 3 capsular polysaccharide in Streptococcus pneumoniae - Enzymatic chain release by an abortive translocation process. J Biol Chem 275:25972-25978.

Guirard BM, Snell EE (1962) Nutritional requirements of microorganisms (in The Bacteria). A Treatise on Structure and Function, Vol. IV: The Physiology of Growth, Academic Press, New York.

Hofvendahl K, Hahn-Hägerdal B (2000). Factors affecting the fermentative lactic acid production from renewable resources. Enzym Microb Tech 26:87- 107.

Johns MR, Goh L, Oeggerli A (1994) Effect of pH, agitation and aeration on hyaluronic acid production by Stretococcus zooepidemicus. Biotechnol Lett 16:507-512.

Kim JH, Yoo SJ, Oh DK, Kweon YG, Park DW, Lee CH, Gil GH (1996) Selection of a Streptococus equi mutant and optimization of the culture conditions for the production of high molecular weight hyaluronic acid. Enzym Microb Technol 19:440-445.

Liu L, Du G, Chen J, Zhu Y, Wang M, Sun J (2009) Microbial production of low molecular weight hyaluronic acid by adding hydrogen peroxide and ascorbate in batch culture of Streptococcus zooepidemicus. Bioresource Technol 100:362-367.

Liu L, Li YLJ, Du G, Chen J (2011) Microbial production of hyaluronic acid: current state, challenges, and perspectives. Microb Cell Fact 10:99.

Macedo AC, Santana MHA (2012) Hyaluronic acid depolymerization by ascorbate-redox effects on solid state cultivation of Streptococcus zooepidemicus in cashew apple fruit bagasse. World J Microbiol Biotechnol 28:2213-2219.

Nielsen LK (2005) Metabolic Engineering of Hyaluronic Acid Production, http://www.cheque.uq.edu.au/research/bioengineering/research/Metabolic_Engineering/HA.html, accessed in 27/11/2005.

Ogrodowski CS, Hokka CO, Santana MHA (2005) Production of Hyaluronic acid by Streptococcus, The effects of addition of lysozyme and aeration on the formation and the rheological properties of product. Appl Biochem Biotechnol 121:753761.

Oliveira DWF, França IWL, Felix AKN, Martins JJL, Giro MEA, Melo VMM, Gonçalves LRB (2013) Kinetic study of biosurfactant production by Bacillus subtilis LAMI005 grown in clarified cashew apple juice. Colloid Surface B 98:518443.

Patil KP, Kamalja KK, Chaudhari, BL (2011) Optimization of medium components for hyaluronic acid production by Streptococcus zooepidemicus MTCC 3523 using a statistical approach. Carbohyd Polym 86:1573-1577 
Pires AMB, Macedo AC, Eguchi SY, Santana MHA (2010a) Microbial production of hyaluronic acid from agricultural resource derivatives. Bioresource Technol 101:6506-6509.

Pires AMB, Santana MHA (2010) Metabolic effects of the initial glucose concentration on microbial production of hyaluronic acid. Appl Biochem Biotechnol 162:1751-1761.

Prieto JG, Pulido MM, Zapico J, Molina AJ, Gimeno M, Coronel P, Alvarez AI (2005) Comparative study of hyaluronic derivatives, rheological behaviour, mechanical and chemical degradation. Int J Biol Macromol 35:63-69.

Rocha MVP, Souza MCM, Benedito SCL, Bezerra MS, Macedo GR, Pinto GAS, Gonçalves LRB (2007) Production of Biosurfactant by Pseudomonas aeruginosa Grown on Cashew Apple Juice. Appl Biochem Biotechnol 136:185-194.

Sancho SO, Maia GA, Figueiredo RW, Rodrigues S, Sousa PHM (2007) Alterações químicas e físico-químicas no processamento de suco de caju (Anacardium occidentale L.). Cienc Tecnol Aliment 27:878-882.
Soltés L, Mendichi R, Kogan G, Schiller J, Stankovská M, Arnhold J (2006) Degradative action of reactive oxygen species on hyaluronan. Biomacromolecules 7:659-668.

Somogyi M (1952). Notes on sugar determination. J Biol Chem 195:19-23.

Stangohl S. 2000. Methods and means for the production of hyaluronic acid. US Patent 6,090,596.

Swann D, Sullivan B, Jamieson G. 1990. Biosynthesis of hyaluronic acid. U.S. Pat. 4,897,349.

Yamada T, Kawasaki T (2005) Microbial synthesis of hyaluronan and chitin: new approaches. J Biosci Bioeng 99:521-528.

Zhang J, Ding X, Yang L, Kong Z (2006). A serum-free medium for colony growth and hyaluronic acid production by Streptococcus zooepidemicus NJUST01. Appl Microbiol Biotechnol 72:168-172.

All the content of the journal, except where otherwise noted, is licensed under a Creative Commons License CC BY-NC. 